\title{
Stirling's Series Made Easy
}

\section{Chris Impens}

1. INTRODUCTION. Stirling's formula for factorials deals with the behaviour of the sequence

$$
r_{n}:=\ln \frac{n ! e^{n}}{\sqrt{2 \pi} n^{n+\frac{1}{2}}} \quad(n=1,2, \ldots)
$$

Its qualitative form simply states that

$$
\lim _{n \rightarrow+\infty} r_{n}=0 .
$$

Quantitative forms, of which there are many, give upper and lower estimates for $r_{n}$. As for precision, nothing beats Stirling's series. This divergent series can be written as

$$
r_{n} \simeq \frac{A}{n}-\frac{B}{n^{3}}+\frac{C}{n^{5}}-\frac{D}{n^{7}}+\cdots,
$$

( $A, B, C, D, \ldots$ are positive constants), where the symbol $\simeq$ means that $r_{n}$ lies between any two successive partial sums of the series. Usually (as in [1, pp. 529-534]) this formula is deduced from Euler's summation formula. In this note we show how to compute in succession the constants $A, B, C, \ldots$ by a very simple technique. Only derivatives and limits of $\mathrm{ln}$ and of rational functions are involved, plus the following elementary property: if $f(x)$ is stricty decreasing (respectively, increasing) for $x>0$ and $\lim _{x \rightarrow+\infty} f(x)=0$, then $f(x)>0$ (respectively, $f(x)<0$ ) for $x>0$. In the sequel we call this property simply $(\mathrm{P})$. It also holds, and will be used, for sequences.

We also show that the constants obtained by this method are the best ones possible, i.e., they cannot be improved by any method whatsoever. In the second section we adapt our method to obtain Stirling's series for the logarithm of the gamma function.

2. STIRLING'S SERIES FOR $\ln (n$ !). We start with a proof of the qualitative form (2), adding monotonicity.

Theorem 1. The sequence $\left\langle r_{n}\right\rangle$ is strictly decreasing and converges to 0 .

Proof. We have

$$
r_{n+1}-r_{n}=1-\left(n+\frac{1}{2}\right) \ln \left(1+\frac{1}{n}\right)
$$

For $\left\langle r_{n}\right\rangle$ to be decreasing, it suffices that $\ln \left(1+\frac{1}{n}\right)-1 /\left(n+\frac{1}{2}\right)$ be positive for all integers $n>0$. The latter is the case if

$$
f(x):=\ln \left(1+\frac{1}{x}\right)-\frac{1}{x+\frac{1}{2}}>0
$$


when $x>0$. Now this is implied by (P): clearly $\lim _{x \rightarrow+\infty} f(x)=0$, and $f^{\prime}(x)=$ $-1 / x(x+1)(2 x+1)^{2}<0$, so $f(x)$ is strictly decreasing on $(0, \infty)$.

Now consider the sequence $e^{r_{n}}, n=1,2, \ldots$ It decreases, because $\left\langle r_{n}\right\rangle$ decreases; being bounded below by zero, it is convergent. It is well known how to deduce from Wallis's formula that its limit is 1 . Hence $r_{n} \rightarrow 0$.

Corollary 1. For all integers $n>0$ it is true that $r_{n}>0$, and this lower bound cannot be improved.

\section{The construction.}

Step 1. Determine $A>0$ such that $0<r_{n}<A / n$ for all integers $n>0$.

As $r_{n}$ converges to zero, the same holds for $a_{n}:=r_{n}-A / n$. By (P), any $A>0$ that makes $a_{n}$ strictly increasing as a function of $n$ will satisfy our requirement. It suffices that

$$
a_{n+1}-a_{n}=1-\left(n+\frac{1}{2}\right) \ln \left(1+\frac{1}{n}\right)+A\left(\frac{1}{n}-\frac{1}{n+1}\right)>0
$$

hold for all integers $n>0$, which happens if

$$
g(x):=\frac{1}{x+\frac{1}{2}}-\ln \left(1+\frac{1}{x}\right)+A \frac{\frac{1}{x}-\frac{1}{x+1}}{x+\frac{1}{2}}>0
$$

for $x>0$. Plainly, $g(x)$ converges to zero as $x \rightarrow+\infty$. By (P), any $A>0$ that makes $g(x)$ strictly decreasing will serve our purposes. Inspecting

$$
g^{\prime}(x)=\frac{x(1+x)-A\left(12 x^{2}+12 x+2\right)}{x^{2}(x+1)^{2}(2 x+1)^{2}},
$$

we see that it suffices to choose $A$ such that

$$
A>\frac{x(x+1)}{12 x^{2}+12 x+2} .
$$

For $x>0$ the right-hand side of (4) has derivative

$$
\frac{2 x+1}{2\left(6 x^{2}+6 x+1\right)^{2}}>0 \text {. }
$$

Therefore the right-hand side in (4) increases to $1 / 12$. Consequently, the best choice by this method is $A=1 / 12$.

Corollary 2. For all integers $n>0$ it is true that $0<r_{n}<1 / 12 n$, and the constant 1/12 cannot be improved.

Proof. Replace $A=1 / 12$ with some $A^{\prime}$ such that $0<A^{\prime}<1 / 12$. We know that the right-hand side of (4) increases to $1 / 12$. Hence $A^{\prime}$ is smaller than the right-hand side for all sufficiently large $x$, say for $x \geq X$. This implies that $g^{\prime}(x)>0$ for $x>X$. Then by (P) $g(x)<0$ for $x>X$, and $a_{n+1}-a_{n}<0$ from some index $n=N$ on. Again by (P), $a_{n}>0$ for $n>N$, and finally $r_{n}>A^{\prime} / n$ for $n>N$. 
Step 2. Determine $B>0$ such that

$$
\frac{1}{12 n}-\frac{B}{n^{3}}<r_{n}<\frac{1}{12 n}
$$

for all integers $n>0$.

Because $r_{n} \rightarrow 0$, the same holds for

$$
b_{n}:=r_{n}-\frac{1}{12 n}+\frac{B}{n^{3}} .
$$

By $(\mathrm{P})$, any $B>0$ that makes $\left\langle b_{n}\right\rangle$ strictly decreasing will satisfy our requirement. It is enough to pick $B$ so that

$$
\begin{aligned}
b_{n+1}-b_{n}= & -\left(n+\frac{1}{2}\right) \ln \left(1+\frac{1}{n}\right) \\
& +\frac{1}{12}\left(\frac{1}{n}-\frac{1}{n+1}\right)-B\left(\frac{1}{n^{3}}-\frac{1}{(n+1)^{3}}\right)<0
\end{aligned}
$$

for all integers $n>0$, and this is the case if

$$
h(x):=\frac{1}{x+\frac{1}{2}}-\ln \left(1+\frac{1}{x}\right)+\frac{1}{12} \frac{\frac{1}{x}-\frac{1}{x+1}}{x+\frac{1}{2}}-B \frac{\frac{1}{x^{3}}-\frac{1}{(1+x)^{3}}}{x+\frac{1}{2}}<0
$$

for $x>0$. Again, $h(x)$ converges to zero for $x \rightarrow+\infty$. By (P), any $B>0$ that makes $h(x)$ strictly increasing will work. Because

$$
h^{\prime}(x)=-\frac{x^{2}(1+x)^{2}-12 B\left(30 x^{4}+60 x^{3}+50 x^{2}+20 x+3\right)}{6 x^{4}(1+x)^{4}(2 x+1)^{2}},
$$

it is sufficient to choose

$$
B>\frac{x^{2}(x+1)^{2}}{12\left(30 x^{4}+60 x^{3}+50 x^{2}+20 x+3\right)} .
$$

The right-hand side increases to $1 / 360$, its derivative being

$$
\frac{x\left(20 x^{4}+50 x^{3}+46 x^{2}+19 x+3\right)}{6\left(30 x^{4}+60 x^{3}+50 x^{2}+20 x+3\right)^{2}}>0
$$

for $x>0$. Hence our method yields $B=1 / 360$ as best choice. As in the previous step, one deduces:

Corollary 3. For all integers $n>0$ it is true that

$$
\frac{1}{12 n}-\frac{1}{360 n^{3}}<r_{n}<\frac{1}{12 n}
$$

and the constant $1 / 360$ cannot be improved. 
Continuing in this manner, one proves that

$$
r_{n} \simeq \frac{1}{12 n}-\frac{1}{360 n^{3}}+\frac{1}{1260 n^{5}}-\frac{1}{1680 n^{7}}+\frac{1}{1188 n^{9}}-\cdots .
$$

All that is required is patience in calculating elementary derivatives (or some elementary computer algebra). The inequalities expressed by the notation

$$
r_{n} \simeq \frac{1}{12 n}-\frac{1}{360 n^{3}}+\cdots
$$

are

$$
\frac{1}{12 n}-\frac{1}{360 n^{3}}<r_{n}<\frac{1}{12 n}
$$

The second of these inequalities was obtained in [6] and the first one appeared in [4], derived by a modification of the argument in [6]. By means of

$$
\frac{1}{12 n+1}<\frac{1}{12 n+\frac{3}{4 n+2}}<\frac{1}{12 n}-\frac{1}{360 n^{3}} \quad(n=1,2, \ldots)
$$

(trivially verified) the first inequality in (8) can be weakened in two steps. Each one of the corresponding lower estimates for $r_{n}$ has appeared in this MONTHLY, the one with the smallest lower bound in [6], the sharper one in [2]. In [3] an inequality is used that amounts to adding a negative term to the minorant of (8).

Here, as we have shown, a general method applies to the whole set of inequalities expressed by the series (7). Thus the partial sum

$$
r_{n} \simeq \frac{1}{12 n}-\frac{1}{360 n^{3}}+\frac{1}{1260 n^{5}}-\cdots
$$

is equivalent to

$$
\frac{1}{12 n}-\frac{1}{360 n^{3}}<r_{n}<\frac{1}{12 n}-\frac{1}{360 n^{3}}+\frac{1}{1260 n^{5}} .
$$

By a proof too long to be included here, it can be shown that our elementary method continues to work for all subsequent terms. Moreover, the series so generated is exactly the Stirling series

$$
\frac{B_{2}}{1 \cdot 2 \cdot n}+\frac{B_{4}}{3 \cdot 4 \cdot n^{3}}+\frac{B_{6}}{5 \cdot 6 \cdot n^{5}}+\cdots,
$$

with the so-called Bernoulli numbers $B_{0}, B_{1}, B_{2}, \ldots$ defined by

$$
B_{0}=1, \quad \sum_{i=0}^{n}\left(\begin{array}{l}
n \\
i
\end{array}\right) B_{i}=B_{n} \quad(n=2,3, \ldots) .
$$

This can be seen as follows. The series (9) is known to be the asymptotic development of $r_{n}$ (see [1, p. 530]). By definition, this means that

$$
\begin{gathered}
\lim _{n \rightarrow+\infty} n\left(r_{n}-\frac{B_{2}}{1 \cdot 2 \cdot n}\right)=0, \\
\lim _{n \rightarrow+\infty} n^{3}\left(r_{n}-\frac{B_{2}}{1 \cdot 2 \cdot n}-\frac{B_{4}}{3 \cdot 4 \cdot n^{3}}\right)=0,
\end{gathered}
$$


etc. Hence

$$
\begin{gathered}
\lim _{n \rightarrow+\infty} n r_{n}=\frac{B_{2}}{1 \cdot 2}, \\
\lim _{n \rightarrow+\infty} n^{3}\left(r_{n}-\frac{B_{2}}{1 \cdot 2 \cdot n}\right)=\frac{B_{4}}{3 \cdot 4}
\end{gathered}
$$

etc. On the other hand, our constants $A, B, \ldots$ have the property that for all positive integers $n$

$$
\begin{gathered}
\frac{A}{n}-\frac{B}{n^{3}}<r_{n}<\frac{A}{n}, \\
\frac{A}{n}-\frac{B}{n^{3}}<r_{n}<\frac{A}{n}-\frac{B}{n^{3}}+\frac{C}{n^{5}},
\end{gathered}
$$

etc. These inequalities imply that

$$
\begin{gathered}
\lim _{n \rightarrow+\infty} n r_{n}=A, \\
\lim _{n \rightarrow+\infty} n^{3}\left(r_{n}-\frac{A}{n}\right)=-B,
\end{gathered}
$$

etc. Comparing (10) and (11) gives $A=B_{2} /(1 \cdot 2), B=-B_{4} /(3 \cdot 4)$, and so forth.

3. STIRLING'S SERIES FOR $\ln (\boldsymbol{\Gamma}(\boldsymbol{x}))$. Generalizing (1), we now put

$$
r(x)=\ln \left(\frac{\Gamma(x) e^{x}}{\sqrt{2 \pi} x^{x-\frac{1}{2}}}\right)
$$

for $x>0$. In [5] a short and direct proof was provided for the assertion that

$$
\lim _{x \rightarrow+\infty} r(x)=0 .
$$

We now show how our method allows one to turn the qualitative formula (13) into a sequence of highly precise upper and lower bounds for $r(x)$. In fact, we can prove that

$$
r(x) \simeq \frac{1}{12 x}-\frac{1}{360 x^{3}}+\frac{1}{1260 x^{5}}-\frac{1}{1680 x^{7}}+\frac{1}{1188 x^{9}}-\cdots
$$

for $x>0$, the coefficients being those of the series (7). By the notation (14) we mean that for $x>0$

$$
\begin{gathered}
0<r(x)<\frac{1}{12 x}, \\
\frac{1}{12 x}-\frac{1}{360 x^{3}}<r(x)<\frac{1}{12 x}, \\
\frac{1}{12 x}-\frac{1}{360 x^{3}}<r(x)<\frac{1}{12 x}-\frac{1}{360 x^{3}}+\frac{1}{1260 x^{5}},
\end{gathered}
$$

etc. To illustrate the reasoning, we establish (15); the proofs are modeled after those of Theorem 1 and step 1 in the previous section. For any $x_{0}>0$, define the sequence

$$
\rho_{n}\left(x_{0}\right)=r\left(x_{0}+n\right) \quad(n=0,1,2, \ldots) .
$$


By (13), the sequence $\left\langle\rho_{n}\left(x_{0}\right)\right\rangle$ converges to zero. It will be strictly decreasing if

$$
\rho_{n+1}\left(x_{0}\right)-\rho_{n}\left(x_{0}\right)=1-\left(x_{0}+n+\frac{1}{2}\right) \ln \left(1+\frac{1}{x_{0}+n}\right)<0
$$

for each integer $n \geq 0$, which would follow if

$$
f(x):=\ln \left(1+\frac{1}{x_{0}+x}\right)-\frac{1}{x_{0}+x+\frac{1}{2}}>0
$$

for every $x>0$. The latter is implied by (P): $\lim _{x \rightarrow+\infty} f(x)=0$ and

$$
f^{\prime}(x)=-\frac{1}{\left(x_{0}+x\right)\left(x_{0}+x+1\right)\left(2 x_{0}+2 x+1\right)^{2}}<0
$$

for $x>0$. So $\left\langle\rho_{n}\left(x_{0}\right)\right\rangle$ is strictly decreasing to zero. By $(\mathrm{P}), \rho_{n}\left(x_{0}\right)>0$ for each nonnegative integer $n>0$; in particular,

$$
\rho_{0}\left(x_{0}\right)=r\left(x_{0}\right)>0 \text {. }
$$

Since $x_{0}>0$ was arbitrary,

$$
r(x)>0 \quad(x>0) .
$$

(Note that our proof does not rely on the fact that $r(x)$ is strictly decreasing on $(0, \infty)$, a property not provided by [5].) To prove the second inequality in (15), define

$$
\alpha_{n}\left(x_{0}\right)=r\left(x_{0}+n\right)-\frac{1}{12\left(x_{0}+n\right)} \quad(n=0,1,2, \ldots)
$$

and proceed as in step 1. It should be clear now how to obtain, by a minor adaptation of the earlier arguments, all the inequalities implicit in (14). Of course, the coefficients are the best possible, since we have proved that they are the best ones even with $x$ restricted to the integers.

\section{REFERENCES}

1. K. Knopp, Theory and Application of Infinite Series, 2nd English ed., Hafner Publishing Company, New York, 1971

2. A. J. Maria, A remark on Stirling's formula, this MonTHLy 72 (1965) 1096-1098.

3. R. Michel, On Stirling's formula, this Monthly 109 (2002) 388-390.

4. T. S. Nanjundiah, Note on Stirling's formula, this Monthly 66 (1959) 701-703.

5. J. M. Patin, A very short proof of Stirling's formula, this MonTHLY 96 (1989) 41-42.

6. H. Robbins, A remark on Stirling's formula, this Monthly 62 (1955) 26-28.

Department of Pure Mathematics and Computer Algebra, University of Ghent, Galglaan 2, B-9000 Ghent (Belgium)

ci@cage.rug.ac.be 